animals - produce characteristic, heritable incubation times and patterns of brain damage. This phenomenon of prion 'strains' continues to produce surprises. When hamster prions were inoculated into mice, the animals lived a long, TSE-free life, and mostly did not accumulate $\operatorname{PrP}^{\mathrm{Sc}}$ in their brains (B. Chesebro, Rocky Mountain Labs, Hamilton, Montana). The injection of $\operatorname{PrP}^{\mathrm{Sc}}$-negative brain extracts from these mice into further mice again resulted in no clinical disease, over a study period of more than 650 days. But when brain extracts from the latter mice were injected into hamsters, the animals died rapidly. So the infectious agent had silently replicated for several years in mice but maintained full virulence towards hamsters. Given that mouse prions are generally harmless to hamsters, it is hard to understand why - in this instance - they retained their infectivity. It would seem that prion strain characteristics dominate over the amino-acid sequence of the prions from the infected host. It is challenging, but maybe not impossible, to reconcile these data with the protein-only prion hypothesis: maybe the strain-specific properties are really encoded in the tertiary or quaternary structure of $\operatorname{PrP}^{S c}$ rather than in its amino-acid (primary) sequence.

At the genetic level, variations in the human prion gene that protect against the development of CJD have disseminated much more efficiently than non-protective variations throughout human populations worldwide (J. Collinge, Inst. Neurology, London $)^{4}$. This provides a compelling case that these protective changes were 'selected for' during the course of evolution - but why would they have been necessary? Collinge suggested that they protected against cannibalism-transmitted prion diseases. He derived the disturbing conclusion that cannibalism was once commonplace among our ancestors, and that prion diseases such as kuru - once a prime cause of death in New Guinea tribes that practised cannibalism - ravaged human populations in the distant past.

On a different note, it was suggested that the concept of prions as proteins that exist in two or more conformations, and replicate by causing other such proteins to change shape, applies not just to those proteins implicated in TSEs (R. Wickner, NIH, Bethesda, Maryland $)^{5}$. Thus, in yeast, heritable traits can be attributable to prions under the following conditions: if they can disappear and later reappear (in the same or a later generation); if their propagation depends on the presence of the gene encoding the protein in question; and if their spontaneous frequency increases upon overexpression of that protein. If one accepts this definition, self-activating enzyme precursors (zymogens) might fall into this category. For instance, yeast proprotease B will self-activate by limited proteolysis (T. Roberts, NIH, Bethesda, Maryland, and R. Wickner). As the activated state is stable and transmissible by transferring the cytosol from one cell to another, it could be regarded as a prion.

Moving into the clinic, we find improvements in the diagnosis of prion diseases. The conformation-dependent immunoassay allows sensitive diagnosis by exploiting the fact that parts of the prion protein become inaccessible to antibodies during conversion to $\operatorname{PrP}^{\mathrm{Sc}}$ (S. Prusiner, Univ. California, San Francisco). This led to the discovery that, in certain circumstances, the disease-associated prion protein is conformationally changed but is not protease-resistant (J. Safar, Univ. California, San Francisco) ${ }^{6}$. So, conformational assays might be inherently more sensitive than the venerable protease-based tests used currently.

But the most startling diagnostic development is the advent of a quantitative, sensitive assay that measures the ability of samples to infect prion-susceptible cells (C. Weissmann, Imperial College, London). The test is inexpensive and can be performed in days, as opposed to conventional infectivity assays in

Animal behaviour

\title{
Homing is a breeze for sea turtles
}

Charles Darwin was one of the first biologists to wonder how migrating green turtles find Ascension Island, a 10-kilometre-long speck of land in the vast mid-Atlantic Ocean (Nature 7, $360 ; 1873)$. These turtles arrive at Ascension Island between December and March, having travelled a daunting 2,200 kilometres or so eastwards from their feeding grounds off Brazil. Graeme C. Hays and colleagues now suggest a role for wind-borne information in this remarkable island-finding ability (Proc. R. Soc. Lond. B doi:10.1098/rspb.2002.2308; 2003).

The navigational feats of marine turtles make them good subjects for researchers interested in migratory behaviour. Early theories proposed that green turtles (Chelonia mydas; pictured) pinpoint Ascension Island by using water-borne odours, carried towards Brazil by the South Atlantic Equatorial Current. More recent laboratory studies have shown that hatchling loggerhead turtles can detect the inclination and intensity of the Earth's magnetic field, perhaps using these features as 'magnetic markers'. However, these promising ideas have yet to be tested on sea turtles in their natural environment.

Hays et al. have now performed just such a test of another theory: that green turtles use wind-borne cues to find their way. The authors found six female turtles that had just nested on Ascension Island, and then 'displaced' them. During the nesting period, persistent tradewinds blow from the southeast. So three of the turtles were transported 50 kilometres upwind of the island, and the other three 50 kilometres downwind. The animals' movements were then tracked by satellite. Female $C$. mydas come ashore to lay eggs several times in a season, so they had a strong urge to return to lay their remaining eggs.

Incredibly, Hays et al. found that the three downwind-displaced turtles returned to the island within 1, 2 and 4 days. Two of those moved upwind eventually returned after 10 and 27 days - but only after passing downwind of the island. A third

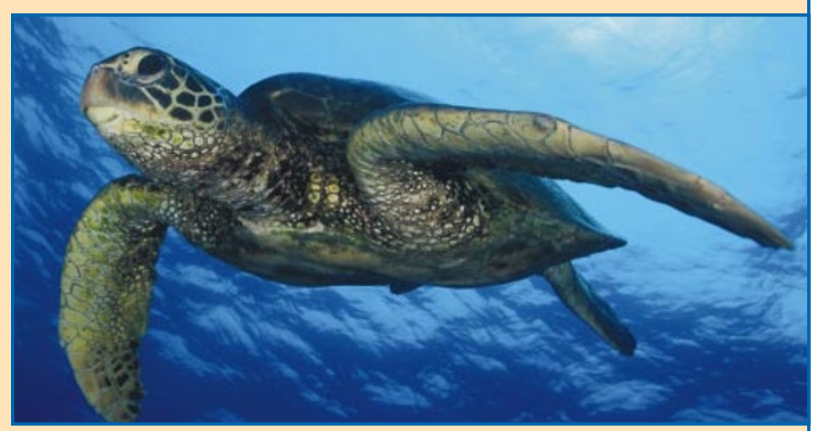

upwind-displaced turtle was unable to locate Ascension Island after 59 days, and was heading back to Brazil when satellite transmissions ceased.

It seems, then, that wind-borne cues emanating from the island perhaps odours or sounds - might direct turtles. The authors concede that such cues will not work accurately over very long distances, so might not on their own account for island finding. But perhaps a combination of mechanisms is at work, with a geomagnetic sense aiding navigation over large scales (hundreds to thousands of kilometres), and smell or hearing operating on scales of tens of kilometres. Other long-distance movers might also use such a scaledependent battery of sensory 'channels' for migration. Identifying the role of particular channels, and when they kick in, will be a challenge, but one that will surely benefit from comparisons of turtles with other vertebrates. David W. Sims David W. Sims is at the Marine Biological Association, The Laboratory, Citadel Hill, Plymouth PL1 2PB, UK. e-mail:dws@mba.ac.uk 